\title{
PLCG2 is differentially expressed in the lymph nodes of patients with metastatic breast cancer.
}

Shahan Mamoor, MS ${ }^{1}$

${ }^{1}$ shahanmamoor@gmail.com

East Islip, NY USA

Metastasis to the brain is a clinical problem in patients with breast cancer ${ }^{1-3}$. Between the breast and the brain reside the secondary lymphoid organ, the lymph nodes. We mined published microarray data ${ }^{4,5}$ to compare primary and metastatic tumor transcriptomes for the discovery of genes associated with metastasis to the lymph nodes in humans with metastatic breast cancer. We found that phospholipase $\mathrm{C}$ gamma 2, PLCG2, was among the genes whose expression was most different in the lymph node metastases of patients with metastatic breast cancer as compared to primary tumors of the breast. Analysis of a separate microarray dataset revealed that PLCG2 was also differentially expressed in brain metastatic tissues. PLCG2 mRNA was present at increased quantities in lymph node metastases as compared to primary tumors of the breast. Importantly, expression of PLCG2 in primary tumors of the breast was correlated with patient recurrence-free survival in lymph node negative breast cancer patients. Modulation of PLCG2 expression may be relevant to the biology by which tumor cells metastasize from the breast to the lymph nodes and the brain in humans with metastatic breast cancer.

Keywords: breast cancer, metastasis, brain metastasis, central nervous system metastasis, lymph node metastasis, phospholipase C gamma 2, PLCG2, systems biology of breast cancer, targeted therapeutics in breast cancer. 
One report described a 34\% incidence of central nervous system metastases in patients treated with trastuzumab for breast cancer ${ }^{2}$. More recently, the NEfERT-T clinical trial ${ }^{6}$ which compared administration of either neratinib or trastuzumab in conjunction with paclitaxel demonstrated that in a randomized, controlled setting, in breast cancer patients treated with neratinib, not only was the incidence of central nervous system recurrence significantly lower, the time to central nervous system metastasis was significantly delayed as compared to patients administered trastuzumab ${ }^{6}$. The alarmingly high rate of central nervous system metastasis described, as well as data, both anecdotal ${ }^{2}$ and from a randomized, controlled setting ${ }^{6}$ illustrating that treatment with trastuzumab may be associated with these events demands an enhanced understanding of the transcriptional makeup of brain and lymph node metastatic tissues to support identification of therapeutic targets, whether they are treatment related or not. The lymph nodes reside between the breast and the brain. We performed a global comparative analysis of lymph node metastatic tumor tissues in patients with metastatic breast cancer, compared to primary tumors and normal breast tissues ${ }^{4,5}$. We discovered transcriptome-wide differential expression of the gene encoding phospholipase C gamma 2, PLCG2, in lymph node metastatic tissues of patients with metastatic breast cancer.

\section{$\underline{\text { Methods }}$}

We used datasets GSE10893 ${ }^{4}$ and GSE100534 ${ }^{5}$ for this global differential gene expression analysis of brain metastatic breast cancer in conjunction with GEO2R. GSE10893 was generated using Agilent-011521 Human 1A Microarray G4110A technology with $n=71$ primary breast tumors and $n=7$ lymph node metastases from patients with breast cancer; analysis was performed using platform GPL887. GSE100534 was generated using Affymetrix Human Gene 1.0 ST Array with $n=16$ primary breast tumors and $n=3$ brain metastases from patients metastatic breast cancer; analysis was performed using platform GPL6244. The Benjamini and Hochberg method of $p$-value adjustment was used for ranking of differential expression but raw $p$-values were used to assess statistical significance of global differential expression. Log-transformation of data was auto-detected, and the NCBI generated category of platform annotation was used. A statistical test was performed to evaluate whether PLCG2 gene expression was significantly different between primary tumors of the breast and lymph node metastases in humans with breast cancer using a two-tailed t-test. For Kaplan-Meier survival analysis, we used the Kaplan-Meier plotter online tool ${ }^{7}$ for correlation of PLCG2 mRNA expression levels with recurrence-free survival in $n=1133$ lymph node positive breast cancer patients and $n=2020$ lymph node negative breast cancer patients.

\section{$\underline{\text { Results }}$}

We performed global comparative transcriptome analysis of metastatic tumor tissues of patients with metastatic breast cancer using published microarray data ${ }^{4,5}$ to describe the transcriptional landscape of metastasis in human breast cancer in an unbiased fashion and for the discovery of novel therapeutic targets.

\section{PLCG2 is differentially expressed in the lymph node metastases of patients with metastatic breast cancer.}

Through blind, systems-level analysis of published microarray data ${ }^{4}$, we identified phospholipase C gamma 2, encoded by PLCG2, as a differentially expressed gene in the lymph node metastatic tissues of humans with breast cancer (Table 1). When sorting each of the genes expressed in lymph node metastases based on significance of difference as compared to primary tumors of the breast in patients with breast cancer, PLCG2 ranked 213 out of 18034 total transcripts (Chart 1), equating to 98.8\% differential expression. Differential expression of PLCG2 in the lymph node metastases of patients with metastatic breast cancer was statistically significant (Chart $1 ; p=2.63 \mathrm{e}-03$ ). 
To attempt to validate transcriptome-wide differential expression of PLCG2 in metastatic tissues of patients with metastatic breast cancer, we queried a second microarray dataset ${ }^{5}$, here comparing primary tumors from patients with breast cancer to brain metastases. Again, we identified PLCG2 as a differentially expressed gene in human brain metastatic breast cancer (Chart 2) When sorting each of the genes expressed in brain metastases based on significance of difference as compared to normal breast tissues, PLCG2 ranked 4663 out of 41093 total transcripts (Chart 2), equating to $88.7 \%$ differential expression. Differential expression of PLCG2 in the brain metastases of patients with metastatic breast cancer was statistically significant (Chart $2 ; p=1.38 \mathrm{e}-02$ ). Thus, differential expression of PLCG2, transcriptome-wide, in the metastatic tissues of women with metastatic breast cancer was conserved across two independent microarray datasets. This suggests that PLCG2 differential expression in human metastatic breast cancer is a bona fide biological process.

PLCG2 is expressed at higher levels in the lymph node metastases of patients with metastatic breast cancer.

We obtained exact mRNA expression levels for PLCG2, in primary tumors of the breast and in lymph node metastases of patients with metastatic breast cancer to determine direction and statistical significance of change in PLCG2 expression in lymph node metastatic tissues. We observed significantly increased expression of PLCG2 in the lymph node metastases of patients with breast cancer as compared to primary tumors of the breast: PLCG2 was expressed at $1.44 \pm 0.91$ arbitrary units (AU) in primary tumors of the breast, while it was expressed at $2.55 \pm 0.97 \mathrm{AU}$ in lymph node metastatic tissues (Figure 1). The difference in PLCG2 mRNA levels between primary tumors of the breast and lymph node metastases was statistically significant (Figure $1 ; p=0.003062$ ).

\section{PLCG2 expression is significantly correlated with survival outcomes in human breast cancer.}

We performed Kaplan-Meier survival analysis ${ }^{7}$ in $n=1133$ lymph node positive breast cancer patients and $n=2020$ lymph node negative breast cancer patients, to evaluate whether PLCG2 tumor expression was correlated with survival outcomes in breast cancer, and to understand whether lymph node status influenced correlation of PLCG2 tumor expression with survival outcomes in human breast cancer. We observed correlation between primary tumor expression of PLCG2 and recurrence-free survival (RFS) in lymph node negative patients with breast cancer (Figure 2), in the lower survival quartile. Lymph node negative patients whose primary tumors expressed low levels of PLCG2 possessed median RFS of 60.22 months, while lymph node negative patients whose tumors expressed high levels of PLCG2 possessed median RFS of 107.43 months. This difference in RFS based on PLCG2 tumor expression in patients with lymph node negative breast cancer approached statistical significance (Figure 2, Chart 3; logrank $p$-value: 0.066; hazard ratio: 0.68 (0.46-1.03)). PLCG2 was not correlated with RFS in lymph node positive patients (Figure 2, Chart 3; logrank $p$-value: 0.53; hazard ratio: 0.91 (0.69-1.21)).

Thus, by mining published microarray data ${ }^{4,5}$ in an unbiased and systematic fashion, we identified PLCG2 as among the genes whose expression was most different, transcriptome-wide, in the lymph node metastases of patients with breast cancer, when compared to primary tumors; we observed significantly increased expression of PLCG2 in lymph node metastases as compared to primary tumors of the breast. Further, we found a significant correlation between PLCG2 expression and patient survival outcomes, as recurrence-free survival was significantly greater in patients whose primary tumors expressed higher levels of PLCG2 as compared to patients whose primary tumors expressed lower levels of PLCG2, and this correlation existed in lymph node negative patients but not in lymph node positive patients. 


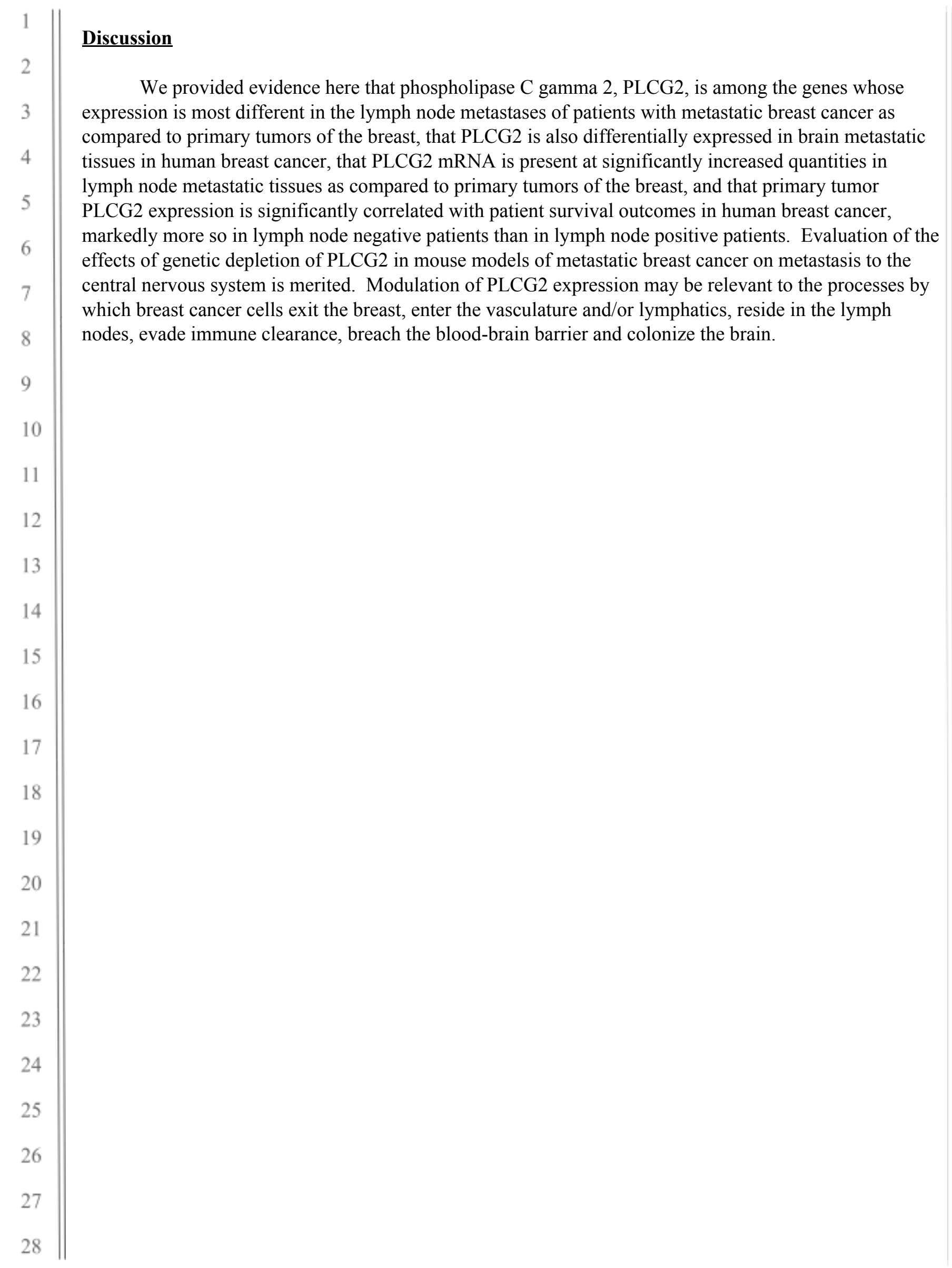




\section{References}

1. Lin, N.U., Amiri-Kordestani, L., Palmieri, D., Liewehr, D.J. and Steeg, P.S., 2013. CNS metastases in breast cancer: old challenge, new frontiers.

2. Bendell, J.C., Domchek, S.M., Burstein, H.J., Harris, L., Younger, J., Kuter, I., Bunnell, C., Rue, M., Gelman, R. and Winer, E., 2003. Central nervous system metastases in women who receive trastuzumab-based therapy for metastatic breast carcinoma. Cancer, 97(12), pp.2972-2977.

3. Tsukada, Y., Fouad, A., Pickren, J.W. and Lane, W.W., 1983. Central nervous system metastasis from breast carcinoma autopsy study. Cancer, 52(12), pp.2349-2354.

4. Weigman, V.J., Chao, H.H., Shabalin, A.A., He, X., Parker, J.S., Nordgard, S.H., Grushko, T., Huo, D., Nwachukwu, C., Nobel, A. and Kristensen, V.N., 2012. Basal-like Breast cancer DNA copy number cancer research and treatment, 133(3), pp.865-880.

5. Schulten, H.J., Bangash, M., Karim, S., Dallol, A., Hussein, D., Merdad, A., Al-Thoubaity, F.K., Al-Maghrabi, J., Jamal, A., Al-Ghamdi, F. and Choudhry, H., 202017. Comprehensive molecular biomarker identification in breast cancer brain metastases. Journal of translational medicine, 15(1), p.269.

6. Awada, A., Colomer, R., Inoue, K., Bondarenko, I., Badwe, R.A., Demetriou, G., Lee, S.C., Mehta, A.O., Kim, S.B., Bachelot, T. and Goswami, C., 2016. Neratinib plus paclitaxel vs trastuzumab plus paclitaxel in previously untreated metastatic ERBB2-positive breast cancer: the NEfERT-T randomized clinical trial. JAMA oncology, 2(12), pp.1557-1564.

7. Györffy, B., Lanczky, A., Eklund, A.C., Denkert, C., Budczies, J., Li, Q. and Szallasi, Z., 2010. An online survival analysis tool to rapidly assess the effect of 22,277 genes on breast cancer prognosis using microarray data of 1,809 patients. Breast cancer research and treatment, 123(3), pp.725-731. 
Rank: 213

Probe ID: 5940

p-value: $2.63 \mathrm{e}-03$

t: -3.1063138

B: -1.6264

Gene: PLCG2

Gene name: phospholipase $\mathrm{C}$ gamma 2

Chart 1: PLCG2 is differentially expressed in lymph node metastatic breast cancer when comparing lymph node metastases to primary tumors of the breast.

The rank of global differential expression, the probe/transcript ID, the $p$-value with respect to differential expression transcriptome-wide, $\mathrm{t}$, a moderated $\mathrm{t}$-statistic, $\mathrm{B}$, the log-odds of differential expression between the groups compared, the gene and gene name are listed in this chart.

Rank: 4663

Probe ID: 7997489

p-value: $1.38 \mathrm{e}-02$

t: -2.6983437

B: -3.302131

Gene: PLCG2

Gene name: phospholipase $\mathrm{C}$ gamma 2

Chart 2: PLCG2 is differentially expressed in brain metastatic breast cancer when comparing brain metastases to primary tumors of the breast.

The rank of global differential expression, the probe/transcript ID, the $p$-value with respect to differential expression transcriptome-wide, $\mathrm{t}$, a moderated t-statistic, $\mathrm{B}$, the log-odds of differential expression between the groups compared, the gene and gene name are listed in this chart. 


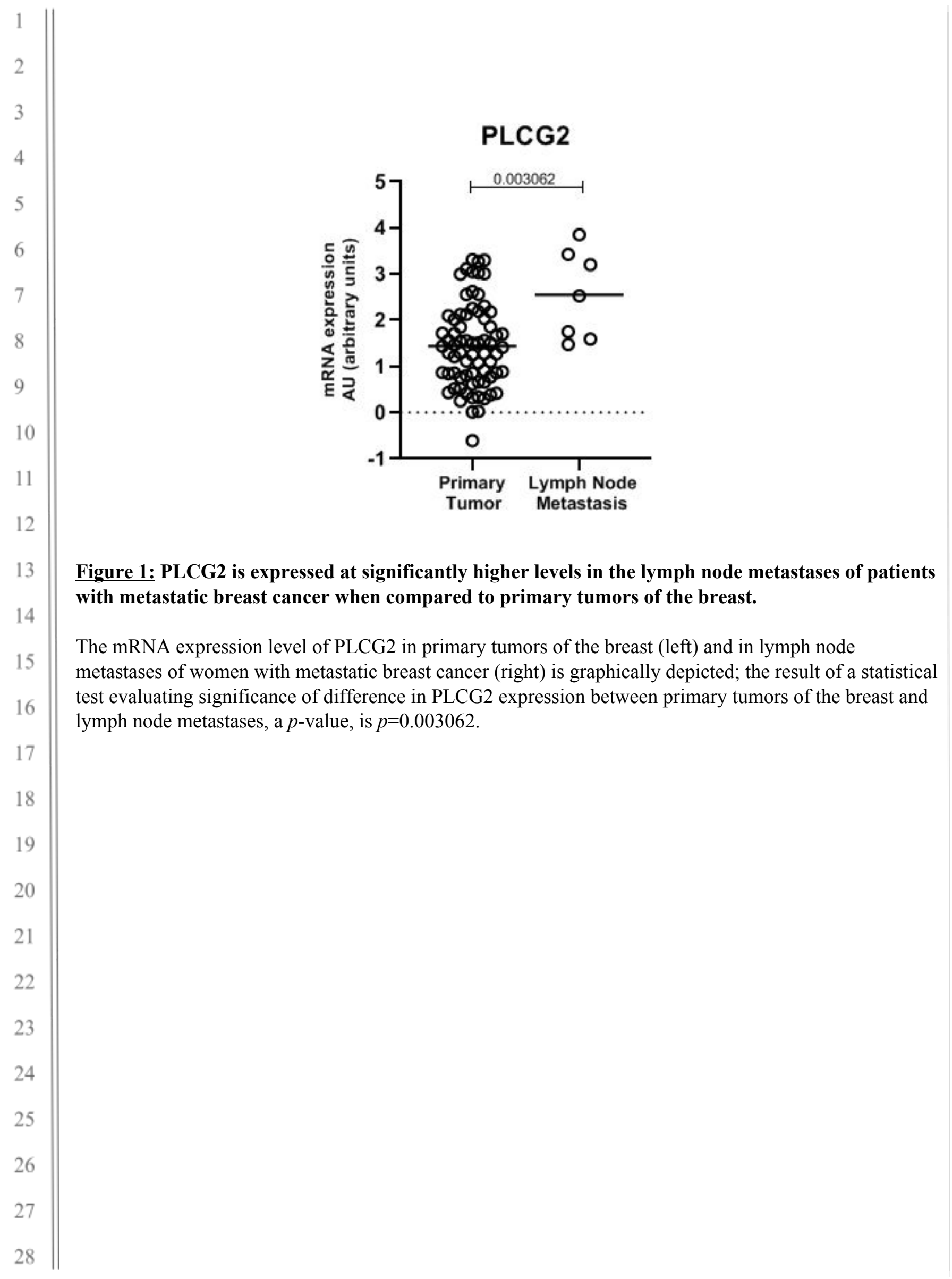



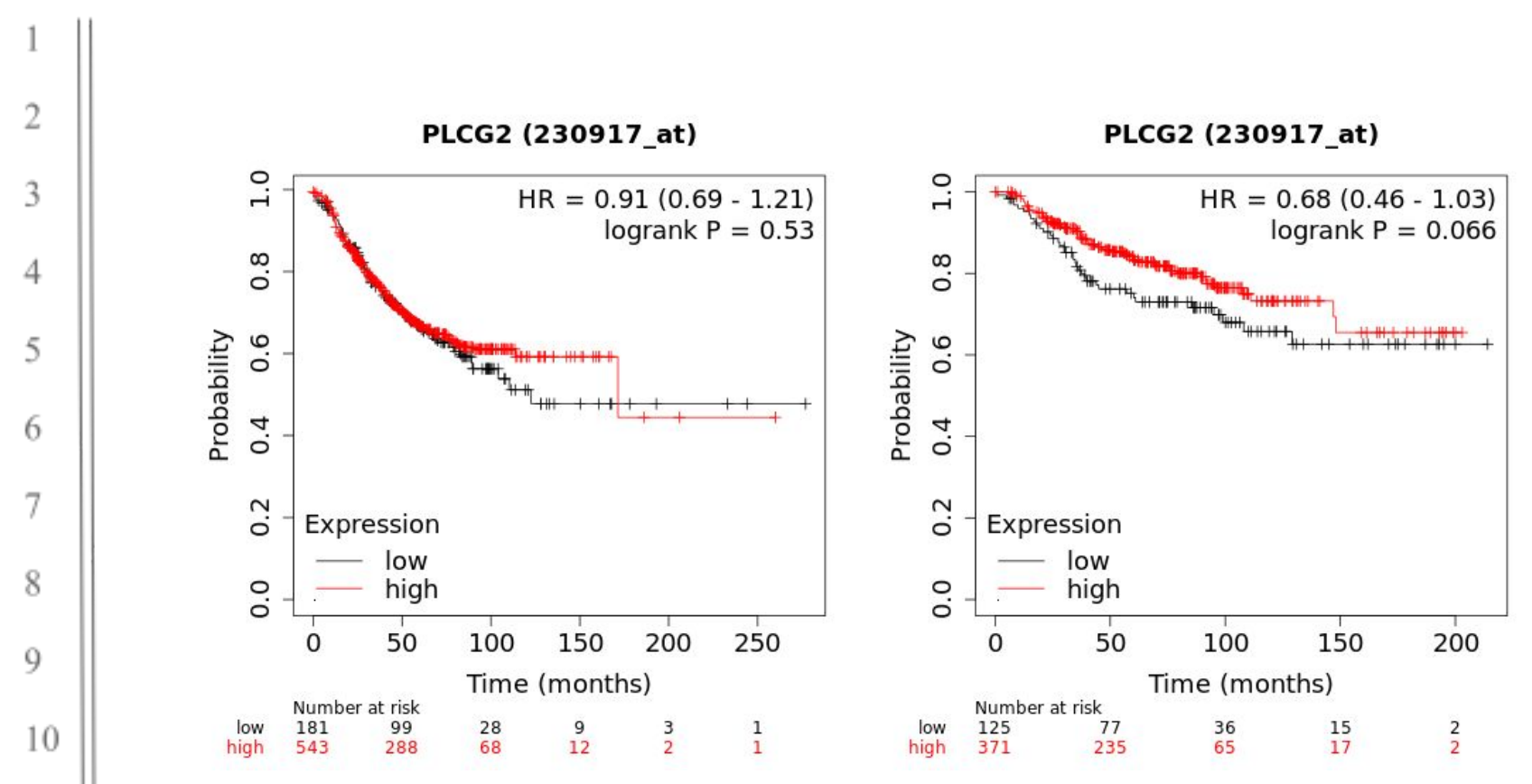

Figure 2: PLCG2 primary tumor expression is correlated with recurrence-free survival in patients with lymph node negative breast cancer patients but not in lymph node positive patients.

Depicted in these Kaplan-Meier plots are the probability of recurrence-free survival in $n=313$ lymph node positive patients (left) and $n=594$ lymph node negative patients (right). The log rank $p$-value denoting statistical significance of difference in recurrence-free survival when comparing the two groups, as well as hazard ratio for this comparison is listed above. Listed below is the number of patients at risk (number of patients alive) per interval, after stratification based on PLCG2 expression; in the first interval, number at risk is number of patients alive; in each subsequent interval, number at risk is the number at risk less those who have expired or are censored. 


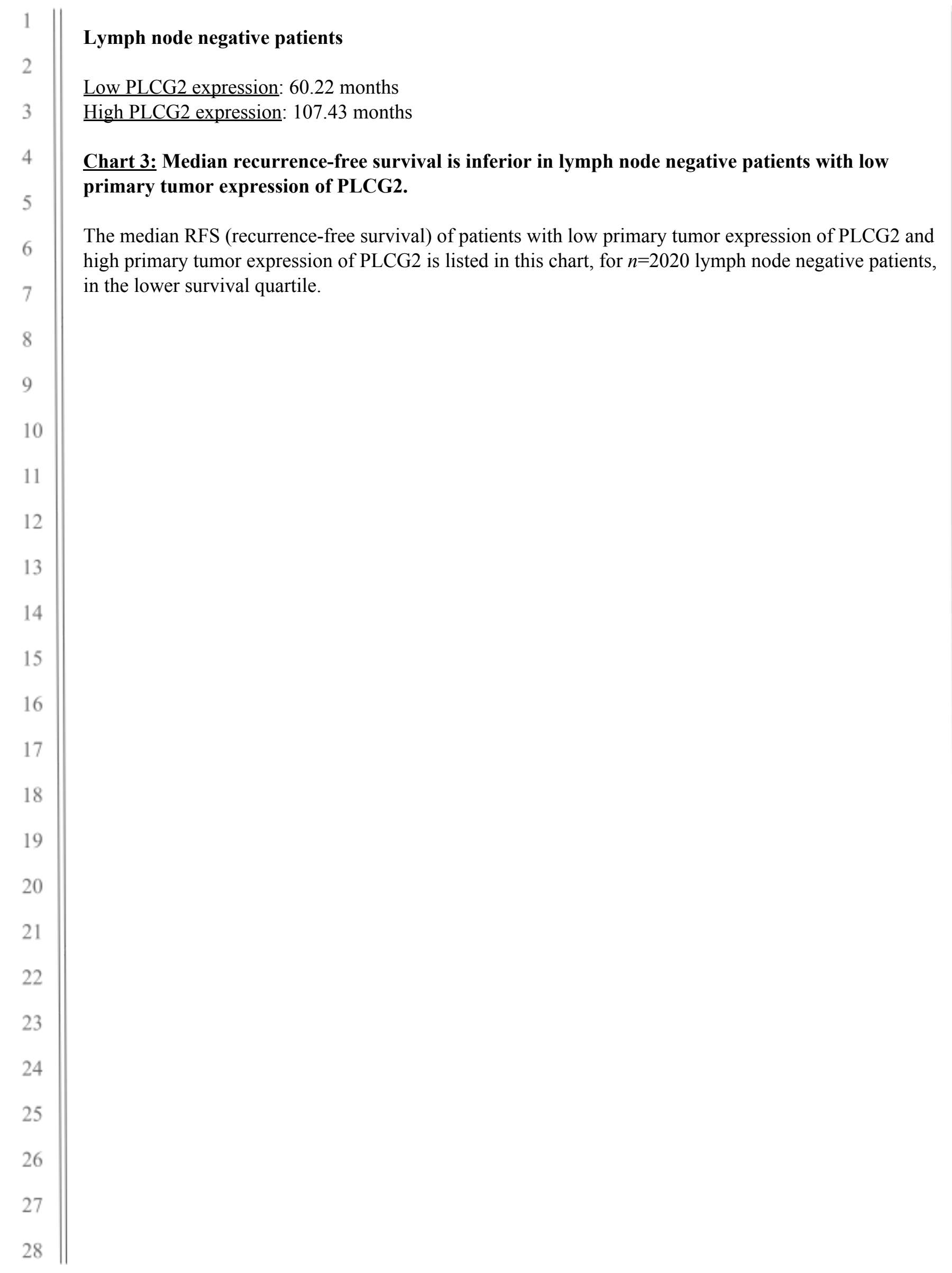

